\title{
Software Solutions to Internet Connectivity in Mobile Ad Hoc Networks
}

\author{
Christer Åhlund ${ }^{1}$, Arkady Zaslavsky ${ }^{2}$ \\ ${ }^{1}$ Luleå University of Technology, Centre for Distance-spanning Technology, Department of \\ Computer Science, SE-971 87 Luleå, Sweden \\ christer@cdt.luth.se \\ ${ }^{2}$ School of Computer Science \& Software Engineering, Monash University, \\ 900 Dandenong Road, Caulfield East, \\ Vic 3145, Melbourne, Australia \\ a.zaslavsky@monash.edu.au
}

\begin{abstract}
In recent years wireless Internet access and wireless communications between peers have become the focus of intensive research efforts in various areas of information and communication technologies. Mobility aspects, software development and support for mobile users are currently of major interest within this research area. The Mobile IP protocol is deployed for mobility management of hosts moving between networks. Ad hoc routing is also of major importance for connectivity between communicating mobile hosts without backbone infrastructure. In this paper we propose and describe an integrated connectivity solution and its software implementation between an ad hoc network running the Ad Hoc On-Demand Distance-Vector Protocol (AODV) and a wired IP network where Mobile IP is used for mobility management. The article also describes a project called Mobile City in which our software solutions and support infrastructure are tested and validated.
\end{abstract}

\section{Introduction}

Current network and distributed systems are unthinkable without sophisticated software solutions that require non-stop evolution and improvement [24, 25]. The IP protocol is the major network protocol used in modern computer networks. This protocol was developed for a wired network topology with stationary routers and hosts. The IP address is used to reach a node in a network (like a router and a host) by determining a route to the destination and identifying the host within the destination network. IP addresses are hierarchically organized with a major network part identifying the network, an optional subnet part identifying a subnetwork within the major network and a host part identifying the host within the network or the subnet. The hierarchical IP address allows routers to only look at the network part when 
finding a route to a destination. The only router looking at the host part of an IP address is the router(s) connected to the network where the destination host resides. To overcome the static network topology problems and to support mobility within IP networks, Mobile IP (MIP)[7] is proposed and partly deployed. MIP can be used for hosts connecting to a foreign network and still function as if they were connected to the home network. Because of the mobility aspects, wireless connections are becoming very popular and are considered the preferred way for a mobile host's (MH) connectivity. Wireless technologies like 802.11a and 802.11b [9] among other technologies are used instead of wired connections and do create a one hop connection to a wired network like Ethernet. To be able to connect to networks equipped with 802.11 access points (AP), the mobile host must be within radio communication range to the AP.

Another type of network becoming popular because of the wireless capabilities in computer communications is an ad hoc network [10]. In ad hoc networks there is no such fixed infrastructure as a wired backbone with routers. Instead, all mobile hosts (MHs), which are ad hoc hosts, usually both work as end user hosts and routers within the ad hoc network. The address space in an ad hoc network enables to work within the ad hoc network regardless of the wired IP network topology. Some ad hoc network proposals make use of clustering to create a hierarchical network scheme, while others use a flat address space. Ad hoc routing protocols can be classified into two major types of routing protocols: proactive and reactive routing protocols [21]. The proactive routing protocols always try to maintain a route to every host in the ad hoc network regardless of whether user data is being sent or not. The proactive approach is the one used in IP networks by routing protocols like RIP and OSPF [13]. In ad hoc networks where mobility might be high, the proactive routing protocols may not be the most optimal protocols to use, since the path created to a destination may be obsolete when the destination is addressed. The reactive routing protocols create a path to a destination when packets need to be sent there. If no user data is sent in the ad hoc network no routes are created. In ad hoc networks reactive routing protocols have been proven to be efficient $[11,12]$.

Our goal with the work described in this article is to enable mobile users to communicate ad hoc when connecting without a backbone, and at the same time have connectivity to a wired IP network infrastructure (the home network or a foreign network) accessing the Internet. When outside communication range to an AP, an intermediate adjacent ad hoc host should be used if possible to reach the AP.

We propose and describe an approach connecting wired IP networks with ad hoc networks running the Ad Hoc On-Demand Distance-Vector Protocol (AODV) [14] for routing in the ad hoc network. Ad hoc hosts will have connectivity with hosts in the ad hoc network as well as in the Internet. For mobility of hosts between networks we use the MIP protocol. Our approach integrates ad hoc networks with IP networks and the Internet.

The contribution of the work proposed and described in this article is an integrated connectivity solution and its prototype software implementation. We propose a new functionality for gateways between IP networks and ad hoc networks for "global connectivity", so that MHs can move between wired IP networks and ad hoc networks while maintaining network connectivity. We also propose protocol changes so that the AODV protocol and the MIP protocol can function together. 
In section 2 we describe the Mobile City project. Section 3 describes our design and implementation. Related work is described in section 4. Section 5 concludes the paper and describes future work.

\section{Mobile City}

Mobile City (www.mobilecity.nu) is an EU funded research and development project that develops both a wireless communication infrastructure and diverse mobile applications that can support various activities of individuals as well as communities. The project involves extensive wireless infrastructure and a number of research initiatives in mobile communications and applications development. The work described in this article is discussed in the context of bringing wireless communication to every person/potential user. The real city in the focus of this project is Skellefteå, located in the northern part of Sweden in the Internet-Bay area. Skellefteå is a typical representative of a regional community in a sparsely populated area of the country (see figure 1), with welldeveloped infrastructure, mostly small and mediumsized enterprises and a broad range of applications that users might need. Rather than focusing on one technology, the project involves a variety of communication and networking-technologies, including 802.11, Bluetooth, GPRS/EDGE, UMTS, etc with each technology supporting a class of applications. In addition, Mobile City addresses the additional important aspect of utilization of mobile

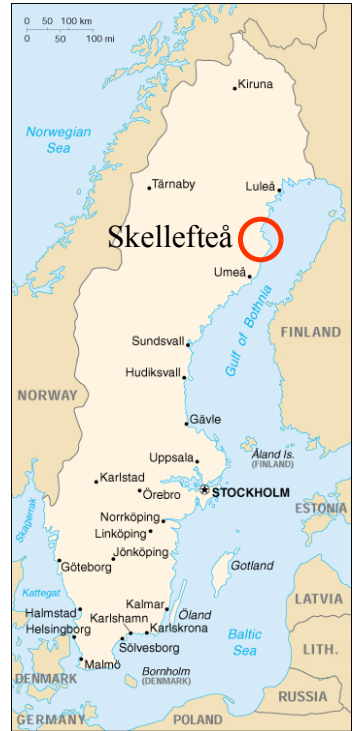

Fig. 1. Regional map Internet-based services.

Current work within Mobile City to support people, companies and business with wireless network connectivity in the community of Skellefteå is described in [18-20].

\section{Connecting IP Networks with Ad hoc Networks}

\subsection{Design considerations}

For ad hoc networks to be integrated with IP networks, ad hoc networks should adapt to the network functionality within IP networks. Often ad hoc networks are seen as self-contained and are of limited size. Ad hoc networks are considered a complementary to IP networks in this article, where Internet connectivity can be 
extended into the ad hoc network, making ad hoc networks a part of the Internet. We assume that an ad hoc network uses a flat address space.

The location of a host in IP networks is identified by the network bits within the IP address (the major network bits and the subnet bits). When a packet arrives at the router connecting the network hosting the destination, and if the destination is connected to a network being a Local Area Network (LAN), the hierarchical IP address is converted to a MAC address using the ARP protocol [15] in IPv4 or the Neighbour Discovery Protocol [3] in IPv6, before the packet is sent in a frame in the last hop using the MAC address as the identifier of the destination. The MAC address represents a flat address space without information about the host's locality within the LAN.

Considering the ad hoc network has a flat address space, it can be seen as a network (major network or subnetwork) within Internet. This will identify an ad hoc network connected to the Internet by its own network number. There is, however, a major difference between a LAN and an ad hoc network. Hosts connected to a LAN are within the same broadcast domain, and are managed as one hop connections by the IP protocol. A packet broadcasted in the LAN will reach all hosts connected to the network. In the ad hoc network a broadcast sent by one ad hoc host may not reach all other hosts. A broadcast need to be retransmitted by hosts in the network so that it will reach all hosts. A broadcast in the ad hoc network running the IP protocol uses the time to live (TTL) value to limit the spreading of a packet. A packet's TTL when arriving at a router connected to a LAN requires a value of 1 to reach a host connected to the network. In the ad hoc network a TTL of 1 when forwarded on the ad hoc network will be discarded after the first hop. This behaviour needs to be managed by gateways connecting ad hoc networks with IP networks.

Instead of using the ARP or the Neighbour Discovery Protocol, the ad hoc routing protocol needs to be used in what is defined as the last hop in IP networks. The functionality in reactive ad hoc routing protocols maps well to the functionality in the ARP protocol and the Neighbour Discovery Protocol, with a request for the host in the last hop and a soft state table. The route request (RREQ) in reactive ad hoc routing protocols can be compared to the ARP request, and the soft state ad hoc routing table to the ARP table.

We assume all MHs in the ad hoc network to have an IP address and to be able to connect to hosts within the Internet. MHs should be able to move between ad hoc networks and still function as if they were connected to the home network. Peers in the ad hoc network should be able to communicate one-hop or multi-hop regardless of their network part in the IP addresses. This means that two hosts homed in different networks will be able to communicate peer-to-peer. MHs homed in an ad hoc network will have the network number given the ad hoc network, and MHs visiting the network will either use their home address as an identifier, an address given by a DHCP server, or if IPv6 is used, stateless auto configuration [3] can be used.

MHs connected to the home ad hoc network will be reached through the IP network by IP routing to the gateway connecting to the ad hoc network. Then the ad hoc routing protocol is used to locate the destination in the ad hoc network. If however the gateway already has an active path to the destination, the packet will be forwarded without a prior RREQ creating a route. 
When an $\mathrm{MH}$ in the ad hoc network addresses a correspondent host, we use the way proxy ARP [15] works to manage where to send a packet for a destination in the same network or in another. In the proxy ARP approach a router looks at the IP address requested, and if the router sees that the destination is in another LAN it responds with its own MAC address. All packets to the IP address will be sent to the router and the router will forward the packet to the destination. The source does not need by itself to investigate if the destination is within the same LAN or not, the router will support this. If the destination is within the same network it will itself respond.

In ad hoc networks we must be able to manage a multi-hop distance between hosts and the gateway, compared to the mechanisms in IP networks with one hop. Instead of the link layer address used in LANs to reach a gateway or a correspondent host in the same network, the IP addresses of the gateway and the MH have to be used, since the gateway may be multiple hops away. If the ad hoc routing protocol uses link layer addresses for routing within the ad hoc network, link layer addresses can be used. But in our work the IP address within the ad hoc network is used to identify MHs and to make route decisions.

To manage mobility of MHs connected to an IP network and trying to connect to different ad hoc networks, MIP is used. In MIP, messages for advertisement, solicitation and registration are sent using local links. For ad hoc networks the MIP messages must be able to travel multiple hops in the ad hoc network. To enhance the performance of our implementation, routes should be installed to the source of the MIP messages.

\subsection{Design}

In our design and implementation described below we make use of MIPv4, and the AODV routing protocol within the ad hoc network.

The design principles described in this section underlie our design considerations discussed in the previous section. We will describe:

- How MIPv4 messages are managed in the ad hoc network.

- How an MH decides what foreign agent (FA) to register with, if it knows several FAs.

- How a destination address from the ad hoc network is found being in the ad hoc network or in the Internet.

For an $\mathrm{MH}$ to be able to communicate ad hoc and to visit foreign ad hoc networks, the MH needs to run both the MIP and the AODV protocols. The same stands for a gateway connecting an ad hoc network to an IP network hosting the AP functionality. For the MIP messages specified to be sent link-local in a LAN, with a TTL value of 1 , we have changed the value to indicate the size of the ad hoc network or the length in hops to an $\mathrm{MH}$.

We have made the AODV protocol MIP-aware to recognize MIP messages so that ad hoc hosts ( $\mathrm{MH}$ and gateway) can install routes based on the messages. Hosts forwarding agent advertisements will install a route to the FA. When an MH then registers with an FA by sending a registration request to the agent, a route will be 
available, without the need to do an explicit RREQ for the FA. The registration request creates a route for the registration reply, and the agent solicitation message creates a route for the unicasted agent advertisement.

An MH discovers a path to a destination by sending an RREQ for the destination. If the destination is known to be outside the ad hoc network, the gateway replies with a proxy RREP (ProxyRREP) to the source. For a destination within the ad hoc network the gateway will function as an ad hoc host forwarding the RREQ.

For a gateway to know which hosts are in the ad hoc network the AODV protocol requires the knowledge of the visitor list in the FA, and all hosts homed in the ad hoc network have to have the same network number as the gateway connecting to the ad hoc network. These requirements are the same as for a LAN in IP networks managing mobility. When a route is requested for a destination with a network number different from the ad hoc network, the visitor list is searched by the AODV process to see if the destination is available in the ad hoc network. If the destination in not within the network the gateway sends a ProxyRREP to the source. If the destination is a foreign $\mathrm{MH}$ visiting the ad hoc network, normal AODV operations are used to discover the destination within the network. Figure 2 illustrates this process. A ProxyRREP is also sent if a destination homed in the ad hoc network is connected to a foreign network. The home agent (HA) functionality used is as specified in MIPv4. Packets coming to the gateway will be processed as shown in figure 3 .

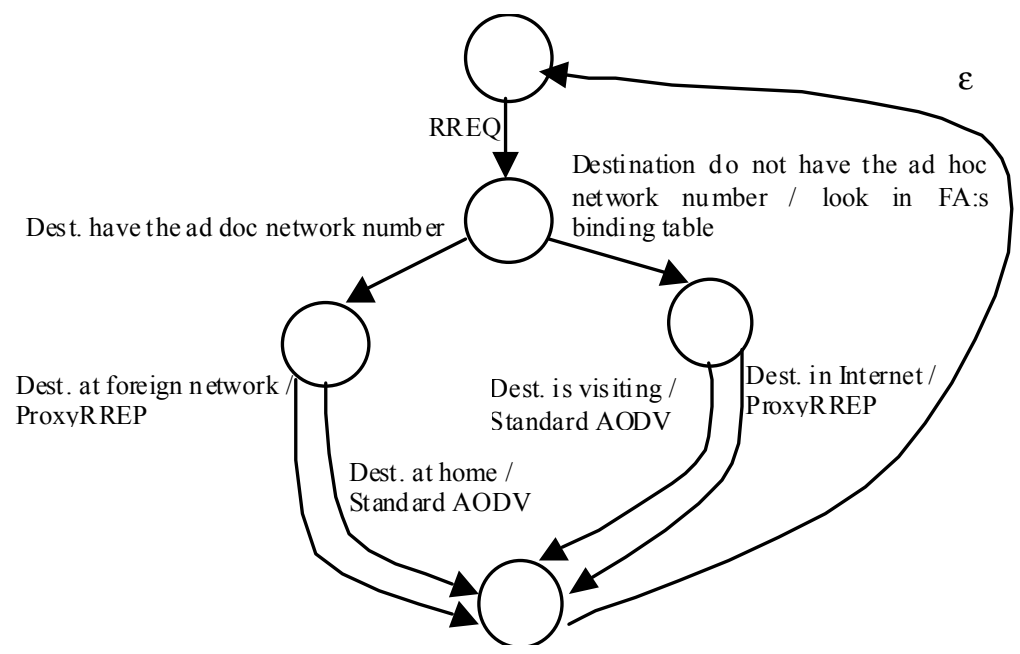

Fig. 2. The process in the gateway to manage AODV RREQ messages 


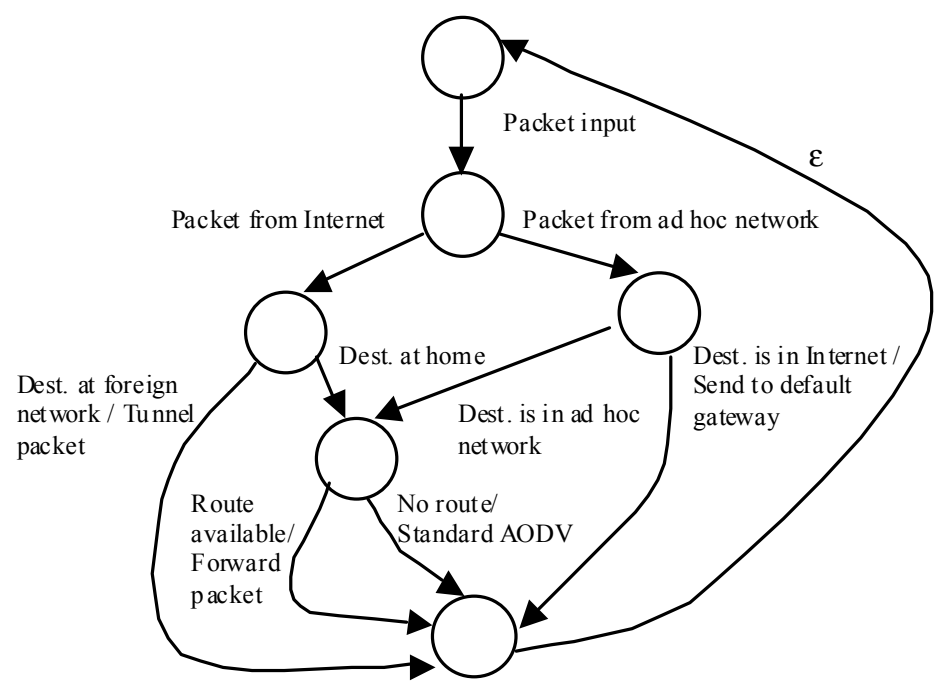

Fig. 3. The flow in the gateway to process an incoming packet

To manage several FAs covering an ad hoc network there is a need to synchronize the visitor information between the FAs. Without synchronization, a gateway may conclude that a destination is within the wired IP network and send a ProxyRREP to the source, while the destination is, in fact, within the ad hoc network but registered with another FA. The FAs synchronize their visitor lists using the wired IP network to offload the ad hoc network, the information is synchronized when an entry is added or deleted from the visitor list in a gateway. In this way, all gateways will be able to see if a visiting host is within the ad hoc network even though it is not registered with the gateway receiving the RREQ. The HA binding cache does not have to be synchronized between the gateways since the gateway responding with a ProxyRREP will be the gateway acting as a HA for the MH connected to a foreign network. Other gateways will believe that the MH is in the ad hoc network.

When several FAs serve an ad hoc network, an MH must be able to choose the best FA to register with. In our approach, the FA closest to the MH will be used based on the roundtrip time (RTT) between the MH and the FA. For each agent advertisement received at an $\mathrm{MH}$, an icmp echo request message is sent to the FA to measure the RTT.

\subsection{Implementation}

The gateway as well as the MHs run the MIP software and the AODV software (see figure 4). The MIP software used is the HUT distribution [16] and the AODV software is a distribution from Uppsala University[17]. The operating system used is Linux 2.4 [22]. 


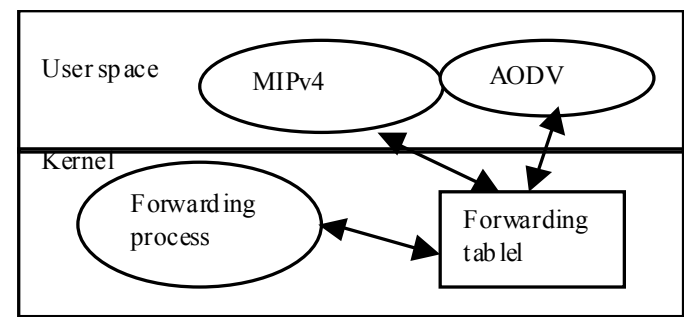

Fig. 4. The processes in a mobile host and their relationship, by using the forwarding table in the kernel.

The MIP and the AODV software operate by modifying the forwarding table within the Linux kernel. In the kernel, the forwarding process forwards the packets using the information in the forwarding table.

The software in the gateway looks as in figure 4 with the extension of a shared memory between the MIP process and the AODV process, so that the AODV process will be informed of visiting MHs. If the gateway hosts an HA as well, the shared memory will also contain the hosts homed in the ad hoc network connected to a foreign network.

To measure the RTT we use the icmp echo request sent to the FA that the $\mathrm{MH}$ knows about. To calculate the metric used to select an FA we use the Jacobson/Karels algorithm [23] for the retransmission timer in the TCP protocol (see formula 1).

$$
\begin{gathered}
\text { Difference }=\text { SampleRTT }- \text { EstimatedRTT. } \\
\text { EstimatedRTT }=\text { EstimatedRTT }+(\delta \times \text { Difference }) . \\
\text { Deviation }=\text { Deviation }+\delta(\mid \text { Difference } \mid- \text { Deviation }) . \\
\text { Metric }=\mu \times \text { EstimatedRTT }+\phi x \text { Deviation. }
\end{gathered}
$$

By using the deviation in the metric calculation the variance will affect the FA selected. A big variance creates imprecise measurements and needs to be considered as a special case.

To switch between FAs the difference between the MeanRTTs must be bigger than a threshold.

In MIP, an MH receiving an agent advertisement assumes the previous link layer sender to be the FA, and uses this address as the default gateway. This must be modified to function in ad hoc networks, otherwise the $\mathrm{MH}$ will believe an intermediate host in the ad hoc network forwarding the agent advertisement to be the FA. We reserve the first care-of address field in the agent advertisement to the FA address within the ad hoc network (see figure 5). The MH can then discover the address of an FA being multiple hops away. 


\begin{tabular}{|c|c|c|c|}
\hline type & length & \multicolumn{2}{|c|}{ sequence number } \\
\hline \multicolumn{2}{|c|}{ registration lifetime } & R B H F M G V & reserved \\
\hline \multicolumn{4}{|c|}{ foreign agent address on the ad hoc network } \\
\hline
\end{tabular}

Fig. 5. The modified agent advertisement extension for the ad hoc network

The agent solicitation message is also modified to function in the ad hoc network for the same reason as the agent advertisement. We have also added a sequence number to limit the broadcast of the solicitation and for the creation of a route to the sender. The solicitation message used in the standard MIP is the same as the router solicitation message. The extension in figure 6 shows the agent solicitation extension added to the router solicitation.

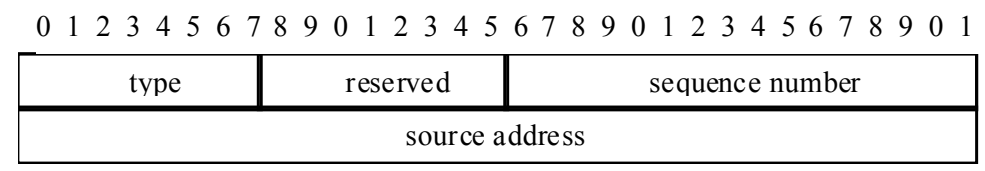

Fig. 6. The agent solicitation extension added to the router solicitation

Since we create a route based on the registration request as well to enhance the performance, we use an extension field in the message to add the source address.

In today's 802.11 connectivity solutions, a host registers with an AP using a PCcard configured in infrastructure mode. In this mode the $\mathrm{MH}$ can only associate with one AP at a time. If two hosts want to communicate without a backbone infrastructure, they can do so ad hoc by configuring the PC-card in ad hoc mode. With this standard network interface configurations, it is not possible to communicate with one physical interface in both modes simultaneously. To manage connectivity with several APs as well as MHs in the ad hoc network, all interfaces are used in ad hoc mode.

\subsection{EVALUATION}

We have thus far created some basic measurements to evaluate the performance of a TCP flow and the RTT from a source in the IP network to an MH connected to a foreign ad hoc network being between 1 and 4 hops from the gateway (see figure 7). 


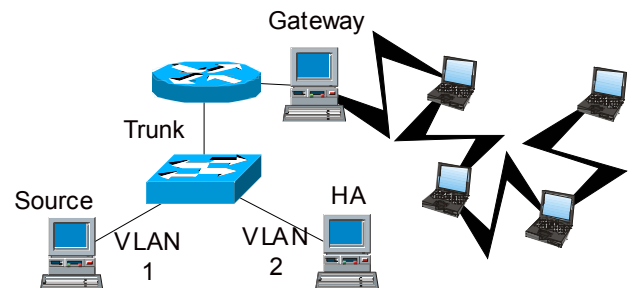

Fig. 7. The topology for our evaluation

The links in the wired IP networks are $100 \mathrm{Mbps}$, and for the wireless connectivity Orinocco $11 \mathrm{Mbps}$ Silver cards are used. The agent advertisement is sent every 5 seconds and the ad hoc route timeout is 10 second. The results are shown in figure 8. All MHs are within 10 meters radius. We filter frames by the MAC-address to control the connectivity between MHs, and to create the ad hoc connections shown in figure 7. The performance degradation in figure 8 is due to the link layer protocol and it shows how an overheard flow will affect the delay and the throughput.
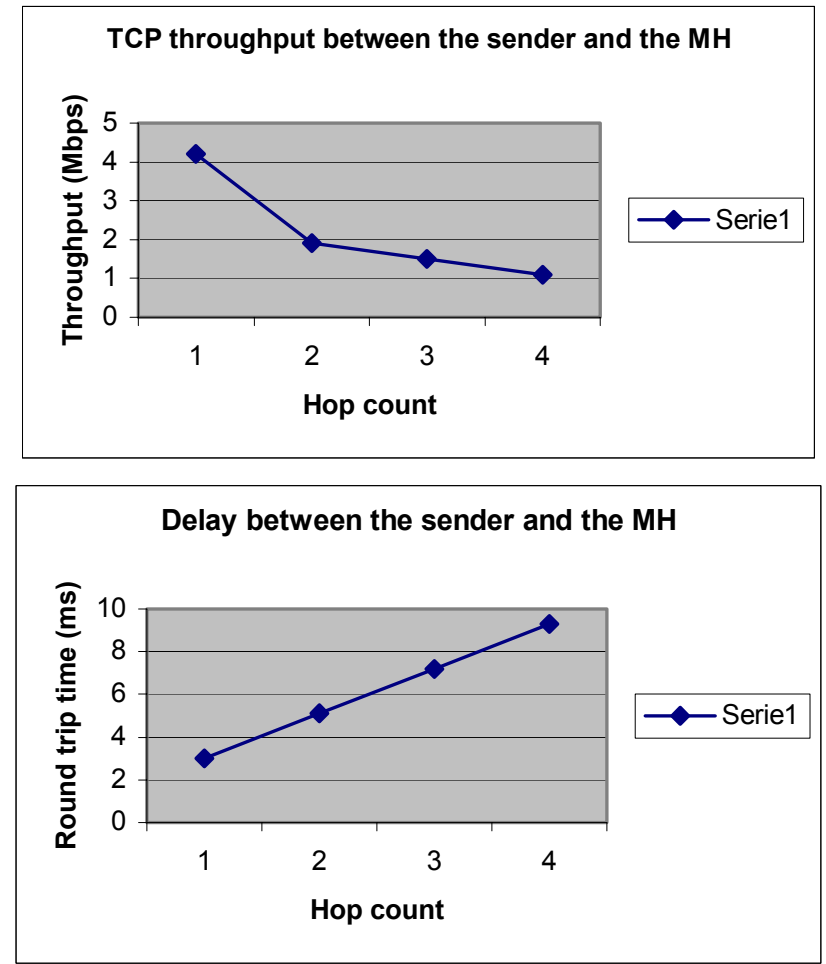

Fig. 8. A basic performance measurement 


\section{Related work}

In [6], authors modify RIP for ad hoc networks and to work with MIP. This approach uses a proactive ad hoc routing protocol, which is not very efficient in ad hoc networks. In [2, 4, 5, 8], a proposal for connections between MIP and AODV is made. In [5] MIPv4 and AODV are connected so that MIP messages will be managed in the ad hoc network. The question of how to select between multiple FAs is not addressed, and an $\mathrm{MH}$ in the ad hoc network has to discover by itself if a destination is within the ad hoc network or not. If the gateway thinks it can receive the destination it replies with an FA RREP (FA-RREP). But before an MH can use the gateway, it first needs to conclude that the destination is not within the ad hoc network and this will delay the connection setup time. MIPv6 management with AODV is proposed in [4] using the neighbour discovery protocol. The same approach for destinations in the IP network is taken as in [5] and it is proposed that router advertisements should not be sent without router solicitation. However, in [2] measurements show that it is more efficient to use the normal MIP behaviour where advertisements are sent without solicitations. In [2] and [8] an approach to selecting between multiple FAs is described, the selection in made on the hop count between the FA and the MH. Hop count may not be the best way to measure what FA to register with since network load is not considered. In [1] MIP and the ad hoc routing protocol DSR are addressed.

A number of software engineering aspects related to wireless and distributed systems are discussed in $[24,25]$.

\section{Conclusion and further work}

In this article we propose and describe an integrated connectivity solution and its implementation connecting IP networks and ad hoc networks running the reactive AODV routing protocol, where MIP is used to manage mobility. The software supports the creation of areas covered by gateways connecting wireless MHs to the Internet. MHs will be able to communicate peer-to-peer or with hosts in the IP network. Our approach proposes a new way to locate a destination inside the ad hoc network or in the IP network, and the selection of a FA based on the RTT between the $\mathrm{MH}$ and the FA.

The solution will support the creation of applications requiring global connectivity. An application using a software socket (TCP or UDP) and one or more network interfaces can rely on the networking software within the MHs and the gateway to find a route to a destination, in the ad hoc network as well as in the Internet. This avoids complexity within the applications.

We are currently running additional performance tests and collecting statistics. We will compare our proposal to select between multiple FA with the proposals in [2] and [8]. The performance and the time to discover a destination in the ad hoc network or in the IP network will be evaluated and compared to the proposal in $[2,4,5,8]$. The evaluation is currently carried out in our network laboratory. The Mobile City project is currently creating a hot spot area in the campus area (se figure 9) where the 
infrastructure can be used for further evaluation in a real environment. The campus hosts two universities among other educational institutions and companies.

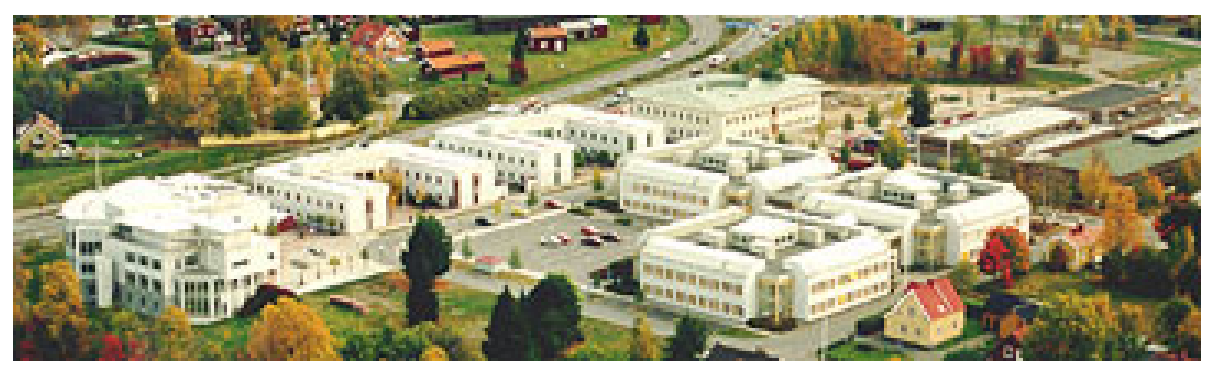

Fig. 9. A hot-spot area - university campus in Skellefteå

\section{References}

1. Broch, J., Maltz, D.A., Johnson, D.B.: Supporting Hierarchy and Heterogeneous Interfaces in Multi-Hop Wireless Ad Hoc Networks. Proceedings of the Workshop on Mobile Computing held in conjunction with the International Symposium on Parallel Architectures, Algorithms, and Networks, IEEE, Perth, Western Australia, (June 1999)

2. Jonsson, U., Alriksson, F., Larsson, T., Johansson, P., Maquire, G.M., Jr.: MIPMANETMobile IP for Mobile Ad Hoc Networks. MOBIHOC (2000) 75-85

3. Thomas, N.: Neighbor Discovery and Stateless Autoconfiguration in IPv6. IEEE Internet Computing. (July 1999) 54-62

4. Wakikawa, R., University, K., Malinen, J.T., Perkins, C.E., Nilsson, A., Tuominen, A.J.: Global connectivity for IPv6 Mobile Ad Hoc Networks. IETF Internet Draft, draftwakikawa-manet-globalv6-01.txt (July 2002)

5. Belding-Royer, E.M., Sun, Y., Perkins, C.E.: Global Connectivity for IPv4 Mobile Ad hoc Networks. IETF Internet Draft, draft-royer-manet-globalv4-00.txt (November 2001)

6. Lei, H., Perkins, C.E.: Ad Hoc Networking with Mobile IP. EPMCC'97 (Oct 1997)

7. Perkins, C.: Mobile IP. IEEE Communications Magazine (May 2002) 66-82

8. Sun, Y., Belding-Royer, E.M., Perkins, C.E.: Internet Connectivity for Ad hoc Mobile Networks. To Appear in International Journal of Wireless Information Networks special issue on "Mobile Ad Hoc Networks (MANETs): Standards, Research, Applications"

9. Gast., M.S.: 802.11 Wireless Networks, The Definitive Guide. O’Reilly (2002)

10. Perkins, C.E.: Ad Hoc Networking. Addison-Wesley (2001)

11. Johansson, P., Larsson, T., Hedman, N., Mielczarek, B. and Degermark. M.: Scenariobased performance analysis of routing protocols for mobile ad-hoc networks. Proceedings of the Fifth Annual International Conference on Mobile Computing and Networking (August 1999)

12. Holland, G., Vaidya, N.: Analysis of TCP Performance over Mobile Ad Hoc Networks. Proceedings of IEEE/ACM MOBICOM (1999) 219-230.

13. RIP, OSPF http://www.cisco.com/univercd/cc/td/doc/cisintwk/ito doc/

14. Perkins, C.E., Belding-Royer, E.M: Ad-hoc On Demand Distance Vector Routing. 2nd IEEE Workshop on Mobile Computing Systems and Applications (1999)

15. Stevens, W.R.: TCP/IP Illustrated, Volume 1: The Protocols. Addison-Wesley (1994) 5364 
16. Dynamics-HUT Mobile IP, http://www.cs.hut.fi/Research/Dynamics/

17. AODV-UU, http://www.docs.uu.se/ henrikl/aodv/

18. Ahlund C., Zaslavsky A.: "Mobile City" Ad Hoc Wireless Network Support for Regional Communities. The Path to 4G Mobility (September 2001)

19. Ahlund C., Zaslavsky A.: Wireless Network Support for Connectivity in Regional Communities. International Conference on Emerging Telecommunications Technologies and Applications, Steps towards the Information Society of the Future, ICETA (2001) 1719

20. Ahlund C., Zaslavsky A., Matskin M.: Supporting Mobile Business Applications in Hot Spot Areas with Pervasive Infrastructure. The First International Conference on Mobile Business, M-BUSINESS (2002)

21. Royer, E., Toh, C.-K.: A Review of Current Routing Protocols for Ad-Hoc Mobile Wireless Networks. IEEE Personal Communications Magazine (April 1999) 46-55

22. Linux, http://www.linux.org

23. Peterson, L.L., Davie, B.: Computer Networks a Systems Approach. Morgan Kaufman Publishert (2000) 391-392

24. Roman, G.-C., Murphy, A. L., Picco, G. P.: A Software Engineering Perspective on Mobility. In A. C. W. Finkelstein, editor, Future of Software Engineering. ACM Press, (2000).

25. Rover, D.T., Waheed, A., Mutka, M.W., Bakic, A.: Software Tools for Complex Distributed Systems: Toward Integrated Tool Environments, IEEE Concurrency, (AprilJune, 1998), 40-54. 